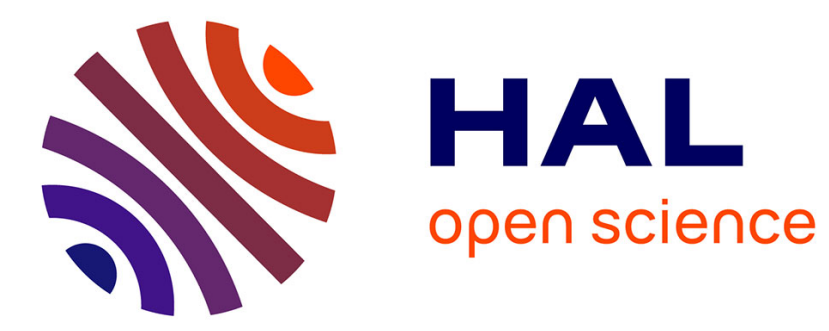

\title{
Fragment de lame d'épée ou de poignard du type de Tréboul-Saint-Brandan du site du Perrou 2 à Maillé (Indre-et-Loire)
}

José Gomez de Soto, Jean-François Chopin

\section{- To cite this version:}

José Gomez de Soto, Jean-François Chopin. Fragment de lame d'épée ou de poignard du type de Tréboul-Saint-Brandan du site du Perrou 2 à Maillé (Indre-et-Loire). Bulletin de la Société préhistorique française, 2014, 111 (3), pp.530-533. halshs-01070925

\section{HAL Id: halshs-01070925 \\ https://shs.hal.science/halshs-01070925}

Submitted on 2 Oct 2014

HAL is a multi-disciplinary open access archive for the deposit and dissemination of scientific research documents, whether they are published or not. The documents may come from teaching and research institutions in France or abroad, or from public or private research centers.
L'archive ouverte pluridisciplinaire HAL, est destinée au dépôt et à la diffusion de documents scientifiques de niveau recherche, publiés ou non, émanant des établissements d'enseignement et de recherche français ou étrangers, des laboratoires publics ou privés. 
Fragment de lame d'épée ou de poignard du type de Tréboul-Saint-Brandan du site Perrou 2 à Maillé (Indre-et-Loire)

\section{Jean-François Chopin \\ José Gomez de Soto}

\section{Contexte de découverte}

Dans le cadre du projet d'aménagement de la ligne ferroviaire à grande vitesse Sud-EstAtlantique, tronçon Tours-Bordeaux, l'opération de fouille menée par l'INRAP à Maillé (Indre-et-Loire), au lieu-dit Perrou, a donné lieu à la découverte d'un fragment de lame d'épée ou de poignard en bronze.

L'opération s'est déroulée du 5 juin au 6 juillet 2012 sur une superficie de $1500 \mathrm{~m}^{2}$, avec pour objectif principal l'étude de vestiges relevant du Néolithique, plus particulièrement du mobilier lithique en silex du Grand-Pressigny. L'emprise de la fouille était située sur la bordure orientale de la vallée de la Vienne, au sein d'un contexte pédosédimentaire alluvial sableux, hydromorphe, lessivé et acide.

L'objet a été repéré au cours d'un décapage mécanique entre $0,35 \mathrm{~m}$ et $0,50 \mathrm{~m}$ de profondeur, en limite d'emprise (fig. 1). Son dégagement a été ensuite réalisé manuellement, dans un rectangle de fouille d'environ $0,30 \mathrm{~m}$ par $0,20 \mathrm{~m}$ de côté pour $0,20 \mathrm{~m}$ de profondeur. Il était situé en position subverticale, légèrement incliné vers l'ouest, pointe vers le haut. Aucune limite de creusement, de différence de texture ou de couleur du sédiment n'a été remarquée lors du décapage mécanique ou de la fouille qui suivit. Le fragment était inclus dans un horizon sédimentaire sableux très lessivé Holocène de mise en place incertaine vu la forte dégradation de ce type de sol (étude géoarchéologique P. Gardères, INRAP). Une gangue d'oxydation assez développée d'environ 19,5 x 6 x 3,5 cm, de formation inhérente au contexte sédimentaire acide et hydromorphe local et tout à fait comparable aux concrétions qui recouvraient les autres objets mis au jour sur ce site, l'enveloppait entièrement (fig. 2, 1).

La nature de l'occupation relative à la présence de cet objet reste incertaine. En premier lieu, il convient de souligner le caractère isolé de cet élément. En effet, aucun autre bronze n'a été trouvé dans les limites de l'emprise. La singularité de cette découverte laisse supposer qu'il a pu être enfoui dans une petite fosse dont les limites ne pouvaient être définies vu le contexte sédimentaire. Par ailleurs, si ce site a connu diverses occupations diachroniques du Néolithique à nos jours, fort peu de vestiges peuvent être chronologiquement parlant rattachés à ce fragment métallique à l'exception d'une céramique au profil biconique, à carène haute et fond plat, décoré d'impressions digitées et unguéales en partie supérieure du corps, attribué avec réserve au Bronze moyen (étude H. Froquet-Uzel, INRAP). Ce vase, mis au jour lors d'un décapage manuel (fig. 1, carroyage 4, ST 1033 et 1034), s'est présenté sous la forme de deux petites concentrations de tessons de chacune environ $0,15 \mathrm{~m}$ de diamètre pour $0,10 \mathrm{~m}$ de profondeur distantes d'environ $0,80 \mathrm{~m}$, qui laissent penser que ces tessons étaient inclus dans deux petits creusements, mais dont la nature reste également indéterminée (trous de piquet, dépôts ?, etc.).

Le fragment de lame a été restauré par M. Biron (chargée de recherche en conservation, INRAP), au musée d'Aquitaine.

\section{Le fragment de lame}


Extrémité distale d'une lame d'épée ou de poignard à section en losange fracturée par flexion, aux tranchants affûtés par martelage, portant un décorée de faisceaux de filets incisés parallèles, puis convergents et réunis à $122 \mathrm{~mm}$ de la pointe dans son état actuel (émoussée). De $179 \mathrm{~mm}$ de longueur actuelle, sa largeur à la cassure était de l'ordre de $30 \mathrm{~mm}$, son épaisseur de $5 \mathrm{~mm}$ (fig. 2, 2).

\section{Attribution typochronologique}

Malgré la disparition de sa partie proximale, il est possible d'attribuer cette lame à l'un des types d'armes d'estoc présentant de façon assez systématique un décor de filets convergents arrêtés à notable distance de la pointe, les épées et poignards du type de Tréboul-SaintBrandan et leurs variantes (Briard, 1965, p. 86 sq. ; Gaucher, Mohen, 1972, fiche 211 ; Gallay, 1988, p. 7 sq. ; Gabillot, 2003, p. 53) ou les épées du type Le Cheylounet (Millotte, 1963 a et b ; Coffyn, 1990 ; Daugas, Vuaillat, 2009).

Les épées des deux types, à grande proximité stylistique (Briard, 1965, p. 103), possèdent une lame effilée à languette curviligne, mais les deux larges échancrures du talon de celles du type Le Cheylounet sont absentes de celles du type de Tréboul. On ne connaît pas de poignards pour le type Le Cheylounet, mais il en existe pour celui de Tréboul. Très proches de ces derniers, offrant le même décor de filets convergents loin de la pointe, sont ceux des types d'Ablon et d'Yville (Gallay, 1988, p. 7-20).

L'absence de la partie proximale de la lame interdirait toute autre précision typologique pour le fragment de Maillé si la répartition géographique des deux familles d'armes n'était éclairante :

- pour les épées du type Le Cheylounet, le sud de la France, sans dépasser au nord la ligne Bec d'Ambés / Jura ;

- pour les épées et poignards des types de Tréboul / Yville / Ablon, Armorique, Normandie, Bassin parisien jusqu'à la zone du confluent Seine-Marne, régions de la Loire moyenne ${ }^{1}$ et inférieure (Briard, 1965 et 1998 ; Gallay, 1988, pl. 62 ; Gabillot, 2003, carte 9-1). Dans les régions de la Loire, on notait les armes de Fontenay-le-Comte, Vendée (Gallay, 1988, ${ }^{\circ} 543$; Cordier, 2009, fig. 148), La Ville-aux-Dames, Indre-et-Loire (Cordier, 2009, fig. 149) et Saint-Ay, Loiret (Gallay, 1988, ${ }^{\circ} 579$; Cordier, 2009, fig. 151), auxquelles on ajoutera désormais le fragment de Maillé. L'épée la plus méridionale connue est celle de SaintCassien, Vienne (Pautreau, 1979, fig. 60 ; Cordier, 2009, fig. 150).

L'origine techno-stylistique des armes des types de Tréboul et apparentés et Le Cheylounet a été largement débattue par ailleurs. Pour les premières, J. Briard (1965, p. 86 et 106) privilégiait une évolution depuis les poignards des tumulus armoricains ; pour les secondes, J.-P. Daugas et D. Vuaillat (2009, p. 118 sq.) mettent en exergue une tradition unéticienne, mais les fortes parentés techno-stylistiques entre les deux productions supposent un complexe jeu d'influences croisées. Leur position chronologique est clairement établie : le Bronze moyen 1 ( $\simeq$ BzB de la chronologie centre-européenne), de 1550 à 1450 environ avant notre 
ère selon les dernières précisions chronologiques disponibles (Gerloff, 2007, p. 124 sq. ; Müller, Lohrke, 2009). Le décor de filets sur la lame de Maillé indiquerait son appartenance à la phase 1 du Bronze moyen 1 ( $\sim$ BzB1), celles de la phase $2(\simeq$ BzB2) n'en portant plus (Gabillot, 2003, p. 119-122).

\section{Interprétation : un dépôt d'un objet unique?}

La position subverticale dans le terrain de la lame du Perrou exclut la perte ou l'abandon sur un sol. Sa fracturation par flexion indique une destruction volontaire, pratique courante pour les dépôts de l'horizon de Tréboul (Briard, 1965). Mais, question : l'isolement de cet objet résulte-t-il d'une perte ou d'un abandon volontaire, autrement-dit, ce bronze constitue-t-il à lui seul un dépôt ? Si l'équivalence bronze isolé = dépôt d'un objet unique a été avancée (Gabillot, 2003), d'autres propositions peuvent aussi rendre compte d'un isolement apparent ou réel (Gabillot, Gomez de Soto, 2007, p. 65). Mais ici, rare cas où l'observation in situ a été possible, l'hypothèse du dépôt paraît bien la plus pertinente, comme pour la hache à talon du dépôt F29 de Ribécourt-Dreslincourt, Oise, du Bronze moyen 2 (Michel, 2013), l'épingle du Bronze final IIIb des Renardières aux Pins, Charente (Boulestin, Gomez de Soto, en préparation) ou probablement l'épée de Pont-sur-Seine, Aube (Boulud, Marillier, 2010).

Les indices d'occupation du site du Perrou pendant le Bronze moyen sont très minces, nous l'avons vu. Quoi qu'il en soit, l'enfouissement d'un dépôt de métal sur un habitat de l'âge du Bronze est bien illustré par ailleurs : par exemple, pour le Bronze moyen, celui du locus B. 119 du Fort-Harrouard à Sorel-Moussel, Eure-et-Loir (Mohen, Bailloud, 1987, p. 199 et pl. 30), pour le Bronze final, ceux de Cannes-Ecluse, Seine-et-Marne (Gaucher, Robert, 1967).

Le même mode d'enfouissement que celui de la lame du Perrou est parfois attesté, par exemple pour les haches du dépôt médocain de Livran à Saint-Germain-d'Esteuil, placées en cercle tranchants en bas (Coffyn, 1972, t. 1, p. 27), ou à Ribécourt-Dreslincourt, le poignard du dépôt F28, enfoui verticalement et sur lequel étaient enfilés six bracelets, et la hache à talon du dépôt F29, placée verticalement tranchant en haut (Michel, 2013, p. 62, 65).

\section{Conclusion}

Dans les régions de la Loire moyenne, en Touraine et en Anjou, les artefacts métalliques du Bronze moyen 1 comportent dépôts tels celui (ou ceux ?) de Pierre-Cou à Chalonnes-surLoire, Maine-et-Loire (Cordier et Gruet, 1985 ; Gabillot et al., 2011) et bronzes isolés : haches à rebords à tranchant étalé ou à ébauche de talon, haches à talon à écusson, pointes de lances du type de Tréboul, épées et poignards des types de Tréboul et apparentés (Cordier, 2009, p. 153-197). L'identité atlantique de cette production est flagrante, mais une meilleure connaissance quant au reste de la culture matérielle pourrait amener à nuancer cette impression: nous y invitent déjà, pour le Bronze moyen $2(\sim \mathrm{BzC})$ les rares céramiques décorées dans le style de la culture des Duffaits (Gomez de Soto, Magne, 2010). 
Le fragment de lame du Perrou constitue ainsi un remarquable document pour la connaissance du début du Bronze moyen en Touraine.

Qui plus est, il offre un cas peu discutable, ce qui n'est pas si commun, de dépôt d'un objet métallique unique, qui plus est enfoui dans des conditions de terrain assez peu fréquentes.

\section{Notes}

1. Le poignard dit de Ceaulmont, Indre (Gallay, 1988, $\mathrm{n}^{\circ}$ 578), est un faux grossier (Girault, 1997).

\section{Références bibliographiques}

MOHEN J.-P., BAILLOUD G. (1987) - La vie quotidienne. Les fouilles du Fort-Harrouard, Paris, Picard (L'Age du Bronze en France 4), 241 p.

BRIARD J. (1965) - Les dépôts bretons et l'Age du Bronze atlantique. Rennes, Laboratoire d'Anthropologie préhistorique, $353 \mathrm{p}$.

BRIARD J. (1998) - Répartition des dépôts de l'âge du Bronze en Bretagne. Ensembles maritimes et ensembles de l'intérieur, in C. Mordant, M. Pernot, V. Rychner (éd.), L'atelier du bronzier en Europe du XXe au VIIIe siècle avant notre ère (actes du coll. int. Bronze'96, Neuchâtel et Dijon, 1996), Paris, CTHS, p. 223-233.

BOULUD S., MARILLIER A. (2010) - Une nouvelle épée du type de Tachlovice découverte à Pont-sur-Seine (Aube), Bulletin de l'Association pour la promotion des recherches sur l'âge du_Bronze, 7, p. 23-26.

COFFYN A. (1972) - Le Bronze final et les débuts du ler Age du Fer autour de l'estuaire girondin, thèse, Université de Bordeaux III, 3 t.

COFFYN A. (1990) - Une épée inédite du Bronze moyen parmi les dessins de F. Daleau, in A. Coffyn (dir.), Aux origines de l'archéologie en Gironde. François Daleau (1845 - 1927), Bordeaux, Société archéologique de Bordeaux, p. 194-199.

CORDIER G. (2009) - L'âge du Bronze dans les pays de la Loire moyenne. Joué-lès-Tours, La Simarre, 702 p.

CORDIER G., GRUET M. (1975) - L'Âge du Bronze et le Premier Âge du Fer en Anjou, Gallia-Préhistoire, 18, p. 157-287.

DAUGAS J.-P., VUAILLAT D. (2009) - Les épées du type "Le Cheylounet" : un aboutissement technologique au Bronze moyen. Hommage à Jacques-Pierre Millotte et Jacques Briard, in A. Richard, A. Daubigney, G. Kaenel, C. Mordant, J.-F. Piningre (dir.), 
L'isthme européen Rhin-Saône-Rhône dans la Protohistoire. Approches nouvelles en hommage à Jacques-Pierre Millotte, actes du colloque de Besançon (16-18 octobre 2006), Besançon, Presses universitaires de Franche-Comté, p. 97-128.

GABILLOT M. (2003) - Dépôts et production métallique du Bronze moyen en France nordorientale. Oxford, British Archaeological Reports, International Series 1174, 471 p.

GABILlOT M., GOMEZ de SOTO J. (2007) - Trésors et cachettes de l'Âge du Bronze en France : cent ans de recherches et d'évolution des méthodes d'analyse, Un siècle de construction du discours scientifique en Préhistoire, actes du XXVIe Congrès préhistorique de France (Avignon, 21-25 septembre 2004), Paris, SPF, vol. II, p. 55-69.

GABILLOT M., SIMON-MILLOT-R., PETIT C. (2011) - Les objets du Bronze moyen récemment découverts à Chalonnes-sur-Loire (Maine-et-Loire). Analyse typologique, environnement culturel et naturel, Bulletin de la Société préhistorique française, 108, 2, p. 331-344.

GALLAY G. (1988) - Die mittel- und spätbronze- sowie älter-eisenzeitlichen Bronzedolche in Frankreich und auf den britischen Kanalinseln, Münich, C.H. Beck (PBF, VI, 7), 202 p.

GAUCHER G., MOHEN J.-P. (1972) - Epées. Paris, SPF (Typologie des objets de l'Age du Bronze en France, I).

GAUCHER G. et ROBERT Y. (1967) - Les dépôts de bronze de Cannes-Ecluses, GalliaPréhistoire, X, p. 169-223.

GERLOFF S. (2007) - Reinecke's ABC and the Chronology of the British Bronze Age, in Chr. Burgess, P. Topping, F. Lynch (éd.), Beyond Stonehenge. Essays on the Bronze Age in honour of Colin Burgess, Oxford, Oxbow Books, p. 117-161.

GIRAULT J.-L. (1997) - Émile Benoist, un dentiste argentonnais « menteur comme un arracheur de dents », Princes et paysans de l'âge du Bronze en Berry, Saint-Marcel, musée archéologique d'Argentomagus, p. 81-86.

GOMEZ de SOTO J., MAGNE P. (2010) - La céramique à décor estampé du Bronze moyen dans les pays de la Loire moyenne. À propos d'un tesson de cruche de Saint-Patrice (Indre-etLoire). Bulletin de la Société préhistorique française, 107, 3, p. 592-594.

MICHEL A. (2013) - Lieu de dépôts multiples du Bronze moyen à Ribécourt-Dreslincourt (Oise), Bulletin de l'Association pour la promotion des recherches sur l'âge du Bronze, 11, p. 61-66.

MILlOTTE J.-P. (1963a) - Le Jura et les Plaines de la Saône aux Ages des Métaux, Les Belles Lettres, Paris, 452 p. 
MILLOTTE J.-P. (1963b) - La place du Massif Central dans la France protohistorique, Bulletin de la Société préhistorique française, LX, p. 661-687.

MÜLLER J., LOHRKE B. (2009) - Neue absolutchronologische Daten für die süddeutsche Hügelgräberbronzezeit, Germania, 87, 1, p. 25-39.

PAUTREAU J.-P. (1979) - Le Chalcolithique et l'Age du Bronze en Poitou (Vendée, DeuxSèvres, Vienne), Poitiers, Centre d'archéologie et d'ethnologie poitevines, $431 \mathrm{p}$.

Jean-François CHOPIN Chargé d'études et de recherches, INRAP Grand Sud-Ouest, ITEM EA 3002, Université de Pau et des Pays de l'Adour jean-francois.chopin@inrap.fr

José GOMEZ de SOTO Directeur de recherche émérite, CNRS

UMR 6566 «CReAAH », Laboratoire Archéosciences, université de Rennes 1 jgzdsoto@free.fr

\section{Légendes}

Fig. 1. Le site Perrou 2 à Maillé (Indre-et-Loire).

Fig. 1. The Perrou 2 settlement in Maillé (Indre-et-Loire, France).

Fig. 2. 1, le fragment de lame du Perrou 2 in situ (photo J.-F. Chopin) ; 2, le fragment de lame après restauration (dessin P. Galibert, INRAP).

Fig. 2. 1, the blade fragment from Le Perrou 2 in situ (photo J.-F. Chopin); 2, the blade fragment restored (drawing P. Galibert, INRAP). 